\title{
The Social Psychology of Education in the Perspective of Positive Psychology
}

\author{
Chunlin $\mathrm{Li}^{\text {a }}$, Weixi Zeng \\ School of Political Science and Public Administration, University of Electronic Science and \\ Technology of China, Chengdu, 611731, China.
}

a182402967@qq.com

Keywords: positive psychology, educational social psychology, practical significance.

\begin{abstract}
Educational social psychology is an emerging edge discipline that originated in the United States in the 1960s and quickly entered the field of education in China. According to the interpretation of this discipline, disciplinary differentiation is a prerequisite for the generation of educational social psychology. Educational practice is the motive force for education social psychology. To explore the subject of educational social psychology from the perspective of positive psychology helps people re-examine the subject of educational social psychology and deepen people's understanding of educational social psychology. This article first elaborates the concept and attribution of educational social psychology, then analyzes the embodiment of positive psychology in educational social psychology, and finally summarizes the practical significance of positive psychology in educational social psychology for the sake of Readers' reference.
\end{abstract}

\section{The Concept and Attribution of Educational Social Psychology}

Educational social psychology is a branch of applied social psychology, and it is also the application of social psychology in education. Educational social psychology is the use of social psychology research methods and theories to study the relationship between people in the process of education. And the resulting psychosocial phenomenon. The educational social psychology is based on education. The early education social psychology takes the psychological phenomenon in school education as the research object. The later educational social psychology develops to a greater extent. Its research field is no longer confined to school education, but extends to school. Unexpected educational activities in education are the common needs of educational practice and social development.

\section{Positive Psychology Reflected in Educational Social Psychology}

The research object of education social psychology is the application of social psychology in education, so this research topic can be divided into: first, the individual psychology in the school, including individual personality traits, behavioral attitudes, etc.; Second, school The group psychology in the group includes teacher groups, student groups, and leadership groups. Third, at the school level, schools are the foundation for the smooth development of educational activities, including social culture, social atmosphere, and other aspects.

Positive psychology pays attention to the research of human positive quality and excavates the inherent potential of human beings to promote the development of individuals and society. If positive psychology is introduced into education, then educational social psychology will show a more dynamic development trend.

\subsection{Positive Psychology Reflects Individual Psychological Aspects of Educational Social Psychology}

The performance of positive psychology in the individual psychological aspects is mainly reflected in the cultivation of individual positive psychological quality and in guiding the individual's active attribution. First of all, from the perspective of cultivating individual's positive psychological quality, positive psychology pays attention to the potential of human beings and pursues a happy life. It is believed that people's advantages are not only confined to overcoming the psychological problems, 
but also have a higher pursuit and will cultivate people's Positive quality is the fundamental goal. Under the guidance of positive psychology, we can use social factors to cultivate students' positive qualities, develop students' sense of responsibility and mission, maintain a good attitude toward society, and become active people with good psychological qualities.

Secondly, from the perspective of guiding the individual's positive attribution, positive psychology advocates actively interpreting the problem and helps the educated to actively analyze their own opinions and problems and make better changes. On the other hand, positive psychology allows individuals to acquire positive meanings, to be more lenient in the face of problems and failures, to be able to derive positive meanings from them, and to be confident that they can continue to progress.

\subsection{Positive Psychology in the Psychology of Educational Social Psychology}

According to the explanation of positive psychology, teachers are also individuals who grow and develop. Teachers and students do not have the opposite relationship between education and education. Teachers also need to cultivate their own positive mentality and treat each student with a positive attitude. Self-growth. Educational psychology requires teachers to treat students with democratic and open mindedness, establish an equal dialogue platform with students, listen to each student's opinions, and not blindly deny and criticize students. On the other hand, teachers should also establish a good emotional relationship with students, impress each student with their true feelings, be good at discovering the merits of students, and expect positive results from students to express themselves better. In classroom teaching and extracurricular development, teachers should be student-centered and transfer the rights to organize, implement, and participate in activities. Students should be the main body of the activity. Teachers should play the role of mentors so that students can truly feel relaxed. With freedom, get exercise and growth at the psychological level.

\subsection{The Positive Psychology Reflected in the Educational Social Psychology School Level}

Positive organizational system theory of positive psychology is very important for individuals to overcome stress and obtain positive psychological quality. However, how contemporary students adapt to such changes is also an important issue faced by active psychological research experts. If society, family, and school can give students enough information and emotional support, then students can more positively evaluate and respond to stressful circumstances, and they can also enhance their sense of security, sense of value, and belonging, and their self-confidence. Self-respect can also be positively responded to help individuals achieve sustained social support and thus be more adaptive to society.

\section{The Practical Significance of Positive Psychology Applied in Educational Social Psychology}

Through the above analysis we can recognize that positive psychology has important manifestations in the individual, group and school level of educational social psychology. This paper believes that the practical significance of positive psychology in educational social psychology mainly includes:

\subsection{Promote the Development of Positive Psychological Qualities of School Members}

Psychology does not focus on individual negative emotions. It does not merely see adverse events in the external world. It is oriented to eliminate various problems in human psychology and bring prosperity to human society. Positive Psychology emphasizes taking a more open-minded view of each person's potential and promoting positive qualities such as happiness and good morals, which is also consistent with the goals of quality education advocated by China. An important component of school education is learning. Educational social psychology extends the factors affecting student learning from the school to the social level. It emphasizes how students use their own positive psychological factors to overcome problems such as school weariness and advocates finding every student in education. The flashlight fosters the enthusiasm of students for active learning and thus generates a strong interest in learning.

\subsection{Promote the Development of School Mental Health Education}

According to positive psychology, everyone is not easy and everyone is imperfect. Therefore, you should actively and objectively treat yourself and others. On the other hand, positive personality includes factors such as diligence, perseverance, and honesty. It is also an important foundation for 
success. Therefore, the basis of quality education in schools is to cultivate students' enthusiasm, and to use positive psychology to promote the improvement of individual students' quality and build more positive attitudes. Interpersonal relationships convey their positive energy to others, including respect, civilization, and motivation.

\subsection{Promote the Construction of a Positive and Complete Education System.}

School education will be strongly influenced by socio-political, economic, and cultural aspects. It will no longer be a closed system. Therefore, schools should be incorporated into the community to solve many complex social and psychological problems faced in school education. Therefore, positive psychology is introduced. It will help integrate various resources in schools, families and society, establish a more complete education system, promote better student growth, and promote the construction of a harmonious society.

\section{References}

[1]. Xu Jiahua, Song Xingchuan. Research Object of Educational Social Psychology [J]. Journal of Hubei Second Teachers College, (2009) No.05, p. 93-95.

[2]. Yan Xiaoyan, Han Xiangming. Analysis of Educational Social Psychology Logical Starting Point [J]. Educational Theory and Practice, (2003) No.03, p. 63-64.

[3]. Yang Yanling. The emergence and development of educational social psychology [J]. Journal of Hebei Normal University (Educational Science Edition), (1999) No. 02, p. 35-38. 